Meta

Journal des traducteurs

Translators' Journal

\title{
Influence comparée de la carte de concepts et du résumé sur la compréhension et la production orales durant l'interprétation consécutive
}

\section{Thi Cuc Phuong Nguyen et François V. Tochon}

Volume 43, numéro 2, juin 1998

URI : https://id.erudit.org/iderudit/003730ar

DOI : https://doi.org/10.7202/003730ar

Aller au sommaire du numéro

Éditeur(s)

Les Presses de l'Université de Montréal

ISSN

0026-0452 (imprimé)

1492-1421 (numérique)

Découvrir la revue

Citer cet article

Phuong Nguyen, T. C. \& Tochon, F. V. (1998). Influence comparée de la carte de concepts et du résumé sur la compréhension et la production orales durant l'interprétation consécutive. Meta, 43(2), 220-235.

https://doi.org/10.7202/003730ar

\section{Résumé de l'article}

Cet article présente l'application de deux techniques d'intervention, la carte de concepts et le résumé, dans le processus d'interprétation consécutive chez les apprentis-interprètes vietnamiens de l'École supérieure des langues étrangères de Hanoi. Se fondant sur une analyse quantitative des interprétations et particulièrement sur une analyse qualitative des entrevues semi-dirigées, les auteurs démontrent les influences tant convergentes que divergentes de la carte de concepts et du résumé sur quatre variables impliquées dans ce processus d'interprétation : la compréhension, la production, la prise de notes et l'état psychologique. Quelques suggestions relatives à l'apprentissage de l'interprétation assistée par la cartographie des concepts sont aussi offertes à la fin de l'article. 


\title{
INFLUENCE COMPARÉE DE LA CARTE DE CONCEPTS ET DU RÉSUMÉ SUR LA COMPRÉHENSION ET LA PRODUCTION ORALES DURANT L'INTERPRETATION CONSÉCUTIVE
}

\author{
THI CUC PHUONG NGUYEN \\ Université de Montréal, Canada \\ FRANÇOIS V. TOCHON \\ Universités de Sherbrooke et de Montréal, Canada
}

\begin{abstract}
Résumé
Cet article présente l'application de deux techniques d'intervention, la carte de concepts et le résumé, dans le processus d'interprétation consécutive chez les apprentis-interprètes vietnamiens de l'École supérieure des langues étrangères de Hanoi. Se fondant sur une analyse quantitative des interprétations et particulièrement sur une analyse qualitative des entrevues semi-dirigées, les auteurs démontrent les influences tant convergentes que divergentes de la carte de concepts et du résumé sur quatre variables impliquées dans ce processus d'interprétation: la compréhension, la production, la prise de notes et l'état psychologique. Quelques suggestions relatives à l'apprentissage de l'interprétation assistée par la cartographie des concepts sont aussi offertes à la fin de l'article.
\end{abstract}

\begin{abstract}
This article discusses two techniques applied in consecutive interpretation by Vietnamese student interpreters at the Hanoi Foreign Languages College: the concept map and the summary. Using a quantitative analysis of interpretations and a qualitative analysis of semidirected interviews, the authors show the convergent and divergent influences which the concept map and the summary have on the four variables of consecutive interpretation process : comprehension, production, note-taking, and psychological state. The article concludes with several suggestions for concept mapping assisted interpretor training.
\end{abstract}

Cet article a pour objectif de présenter les résultats d'une recherche quasi expérimentale réalisée auprès de 61 apprentis-interprètes vietnamiens afin de comparer les influences de la carte de concepts et du résumé sur les processus mentaux (de compréhension et de production) de l'interprétation consécutive ${ }^{1}$. L'article se compose de cinq sections. La première est réservée à la présentation de la carte de concepts (sa forme, sa définition). La deuxième fait référence à une hypothèse relative à l'influence de la carte de concepts sur la compréhension et la production orales propres à l'interprétation consécutive. Dans la troisième section, nous faisons la comparaison entre la carte ${ }^{2}$ et le résumé. Le schéma d'expérimentation et les résultats de la recherche seront traités respectivement dans la quatrième et dans la dernière section.

Meta, XLIII, 2, 1998 


\section{LA CARTE DE CONCEPTS}

\section{Les formes de cartes de concepts}

Les cartes de concepts peuvent prendre des formes variées. Nous répertorions deux types de cartes de formes différentes : la carte de réseau et la carte de points. Parmi les cartes de réseau, on peut trouver les cartes dont la forme ressemble à une toile d'araignée (Buzan 1974) ou encore à un arbre généalogique (Novak et Gowin 1981, 1984; Cliburn 1986, 1990; Breton 1991) ou à un réseau sémantique (Lindsay et Norman 1980; Tardif 1992; Dancette 1992, 1994). Quant aux cartes de points (Trochim 1989; Valentine 1989), on peut rencontrer des graphiques composés de points ou de nuages de points représentant les concepts différents, qui ressemblent à certains types de graphique statistique. Dans notre recherche, nous n'avons retenu que la forme de l'arbre généalogique comme celle de Novak et Gowin (1984). Ce choix est justifié par le fait que c'est la forme la plus utilisée dans les recherches en éducation. De plus, elle est facile à lire avec les concepts encadrés, les flèches nommées et les hiérarchies claires. L'exemple d'une des cartes de concepts utilisées dans notre expérimentation est donné dans l'annexe 1. Dans ces cartes, les concepts encadrés ou encerclés représentent les idées essentielles du texte. Les flèches reflètent les relations entre ces idées. Le rectangle au milieu de la carte indique qu'il s'agit du thème ou du concept principal du texte. Les chiffres sur certaines flèches indiquent l'ordre de présentation des idées selon la structure du texte. Selon la carte traditionnelle, chaque cercle ou rectangle ne contient qu'un seul concept. Dans nos cartes, nous n'avons pas maintenu cette règle pour des raisons d'économie, d'espace et de clarté de la carte. Par exemple, si nous respectons cette tradition, l'entité nominale comme Pensée scientifique $\uparrow$ dans la carte du texte 1 ne devra pas se trouver dans une seule bulle. Elle devrait se diviser en trois bulles reliées entre elles de façon suivante évolution $\rightarrow$ pensée $\rightarrow$ sciences. Mais si toutes les bulles semblables sont divisées en deux ou trois autres bulles avec un nombre de flèches accru, la carte risquera de devenir excessivement complexe, ce qui entraînera évidemment, à notre avis, une difficulté accrue dans la lecture des cartes pour les utilisateurs. Dans nos cartes, chaque bulle représente plutôt une unité informative à traiter. Cette unité peut se composer d'un seul substantif, d'un substantif et ses attributs ou des signes faciles à comprendre comme la flèche $(\uparrow)$ pour exprimer l'augmentation, la croissance ou les barres $\longrightarrow$ pour dire l'élimination ou l'annulation. On peut aussi appeler ces unités informatives «unités de traduction» ou «unités d'interprétation» pour notre recherche. Sur ce point, il faut se rappeler que la notion d'«unité de traduction» est relative puisqu'elle est «inscrite dans un processus individuel, probablement non normalisé» (Dancette 1990 : 123). Ainsi, on peut trouver des cartes variées avec des unités de traitement différentes pour un seul texte. Dans le souci de valider les instruments de recherche utilisés, nos cartes finales ont été faites en nous appuyant sur le consensus de deux experts dans le domaine de la carte conceptuelle.

Nous avons donné une idée de ce qu'est une carte de concepts en ce qui concerne ses variantes formelles, nous proposons maintenant sa définition.

\section{La définition de la carte de concepts}

Il existe bon nombre de définitions de la carte de concepts qui se différencient au niveau de la forme, du contenu ou de l'origine de la carte (Novak et Gowin 1984; Stice et Alvarez 1986; Pankratius et Keith 1987; Naïdu 1990; Tochon 1990). Pour une analyse plus détaillée de ces nuances, voir Nguyen et Tochon (sous presse). La définition finale que nous avons retenue pour la recherche présente est la suivante : une carte de concepts est une représentation graphique hiérarchisée, externe, de l'organisation interne des informations d'un texte ou d'un domaine dans la mémoire d'un sujet.

Nous passons maintenant à l'hypothèse de la recherche. 


\section{L'HYPOTHÈSE DE L'INFLUENCE DE LA CARTE DE CONCEPTS SUR LA COMPRÉHENSION ET LA PRODUCTION ORALES PROPRES À L'INTERPRÉTATION CONSÉCUTIVE}

Dans cette section, nous allons aborder d'abord l'hypothèse susmentionnée et, ensuite, la comparaison de deux techniques d'intervention utilisées dans l'expérimentation : celle de la carte de concepts et celle du résumé.

\section{Hypothèse de recherche}

Une revue des écrits relatifs à plusieurs domaines différents, à savoir la cartographie des concepts, la compréhension et la production langagières orales, la traductologie et, particulièrement, l'interprétation consécutive, nous permet de conclure qu'à l'heure actuelle, les recherches sur les applications de la carte de concepts dans l'interprétation consécutive sont presque inexistantes. Pour cette raison, l'hypothèse d'une telle influence de la carte de concepts a été posée comme résultante d'une déduction logique, faite à partir des résultats de recherches dans les domaines précités. Pour arriver à cette inférence, il nous a fallu relier les trois thèses suivantes :

Thèse 1: La carte de concepts influence la compréhension et la production écrites.

Thèse 2 : Les processus de compréhension et de production écrites sont similaires à ceux de compréhension et de production orales.

Thèse 3 : Les processus de l'interprétation consécutive sont des processus de compréhension et de production orales.

La justification de ces thèses par des recherches dans les domaines des cartes de concepts, de la compréhension-production écrites-orales et en traductologie peut être trouvée dans Nguyen et Tochon (sous presse). Ici, nous les retenons seulement pour montrer grosso modo comment l'hypothèse a été formulée :

SI La carte de concepts influence la compréhension et la production écrites (thèse 1)

et SI Les processus de compréhension et de production écrites sont similaires à ceux de compréhension et de production orales (thèse 2)

ALORS : La carte de concepts peut influencer la compréhension et la production orales

COMME Les processus de l'interprétation consécutive sont des processus de compréhension et de production orales (thèse 3 )

ALORS : La carte de concepts peut influencer la compréhension et la production orales dans l'interprétation consécutive (hypothèse de la recherche).

La démarche qui mène à l'hypothèse ci-dessus est faite dans le souci d'assurer un appui théorique à la recherche, d'autant plus que l'application de la carte de concepts dans la compréhension et la production orales, propres à l'interprétation consécutive, reste encore très rare. Un survol des recherches en traductologie n'a permis de repérer que deux chercheurs utilisant une sorte de cartes de concepts : Dancette $(1990,1992,1994)$ et Gile (1990).

Dancette $(1992,1994)$ a utilisé le réseau sémantique en vue d'une désambiguïsation des passages difficiles du texte à traduire. Ce réseau possède une structure qui se rapproche beaucoup de celle de la carte mais qui reste à un niveau propositionnel assez simple.

Gile (1990) a proposé d'utiliser aussi cette technique afin de résoudre les problèmes de compréhension et de production des énoncés spécialisés en traduction. C'est la recherche de Gile qui se rapproche le plus de la nôtre.

Selon le modèle de Gile, les phrases peuvent être analysées en entités nominales (substantifs ou groupes nominaux) déterminées par des attributs et reliées entre elles par des liens fonctionnels ou logiques comme la causalité, la comparaison... (pour les exemples de ce modèle, voir Gile 1990 : 28-29, figures 1b et 2). À travers le modèle de Gile, on 
reconnaît facilement la forme d'une carte de concepts comme la nôtre avec les entités informatives reliées entre elles. Pour Gile, un tel modèle simplifiait la réalité mais il lui semblait assez représentatif dans son principe de compréhension des énoncés spécialisés ainsi que généraux.

Au niveau de la production, Gile (1990) a réalisé une autre expérimentation dans laquelle il présentait une idée de manière schématique, contenant aussi peu d'éléments verbaux que possible. Plus concrètement, il a présenté aux étudiants en traduction un dessin où figurait un pare-brise de voiture et devant celui-ci, un panneau de circulation sur lequel s'écrivait : Paris, $50 \mathrm{~km}$. Ensuite, il a demandé aux étudiants de communiquer l'information du panneau en langue maternelle. À travers l'analyse des énoncés recueillis, Gile a remarqué une certaine liberté dans la production langagière. La superposition (ou l'organisation hiérarchique) des éléments d'information semblait être une donnée «très fondamentale en matière de production du discours au regard de la communication» (Gile 1990 : 27).

Après avoir montré comment notre hypothèse de recherche a été formulée et après l'avoir soutenue par la recherche de Gile (1990), nous passons maintenant à la troisième section.

\section{LA COMPARAISON DE LA CARTE DE CONCEPTS AVEC RÉSUMÉ}

Dans cette section, nous comparons le contenu et la structure de la carte de concepts à ceux du résumé tout en essayant de relier ces structures aux processus mentaux de la compréhension et de la production langagières.

La carte de concepts, comme nous l'avons définie dans la section 1.2, est une organisation graphique hiérarchisée des informations d'un texte ou d'un domaine. Dans le cadre de cette recherche, il s'agit des informations d'un texte. Celles-ci ne sont pas retenues de façon fortuite mais sélective. Les démarches de construction d'une carte de concepts (Novak et Gowin 1984; Ahlberg 1991; Breton 1991) montrent que seules les idées principales du texte et les relations entre celles-ci sont retenues dans la carte. Les règles les plus utilisées comprennent trois étapes majeures :

- trouver les idées ou concepts principaux représentant le sujet abordé,

- arranger hiérarchiquement ces idées ou concepts selon leur importance et les encadrer,

- relier les idées ou concepts par les flèches et nommer ces relations.

La carte de concepts d'un texte représente ainsi le contenu fondamental ou, autrement dit, la macrostructure du texte, par une structure informative hiérarchisée. La macrostructure d'un texte fait référence à l'essentiel du texte organisé en un tout cohérent (Kintsch et van Dijk 1978). Cependant, il faut reconnaître le caractère relatif de la macrostructure d'un texte. Elle peut s'arrêter au niveau du titre, des sous-titres ou aller plus loin jusqu'aux idées principales de chaque paragraphe du texte. Comme dans le cas de toute activité de compréhension, il n'existe pas de macrostructure unique pour un texte, ce qui exige une fois de plus le compromis des juges dans la création de nos cartes des macrostructures conceptuelles.

Par rapport à la carte, le résumé représente aussi le contenu essentiel ou la macrostructure du texte. Pour créer un résumé, les règles générales sont les suivantes :

- réduire au quart en général,

- dégager les idées principales. Résumer les exemples s'ils sont nombreux,

- respecter la progression du texte,

- rester neutre (ni commentaire, ni critique) (Fournier et Dutertre 1982). 
Cependant, la forme discursive du résumé diffère nettement de celle de la carte. Le résumé représente les informations sous une forme linéaire, celle habituelle du langage, tandis que la carte représente les mêmes informations sous une forme hiérarchique, «assez étrangère dans la quasi-totalité des énoncés informatifs» (Gile 1990 : 27). En d'autres mots, le résumé se compose des phrases entières ou des propositions linéaires tandis que la carte comprend les propositions déjà analysées graphiquement en prédicats et en arguments. Selon Kintsch et van Dijk (1975), un texte, y compris le discours oral, est construit par des propositions exprimées en termes de prédicats et d'arguments. Le prédicat indique le procès (verbe, adjectif, relations logiques...) et l'argument indique l'objet ou l'être. Dans le cas de la carte de concepts, les arguments se trouvent le plus souvent dans les formes géométriques (cercle, rectangle, carré...) et les prédicats sont représentés par les flèches, nommées ou non. Toutefois, selon les formes de cartes, on trouve bien des exceptions à ce principe théorique, en raison de l'enchâssement de prédicats implicites. Il n'y a qu'à se reporter à la carte dans l'annexe 1 pour s'en convaincre.

Quels sont les rôles que peuvent jouer ces structures différentes dans le processus d'interprétation consécutive ? Afin de répondre à cette question, nous faisons d'abord, une très brève présentation des recherches traitant de la nature de ces processus.

Sticht et al. (1974), Denhière (1984), Deschênes (1988) estiment que les processus psychologiques de compréhension, que ce soit à l'écrit ou en oral, sont tous des processus de conceptualisation. La compréhension ne serait pas un processus d'encodage passif des mots ou des phrases. Il s'agirait d'une activité mentale active dans laquelle les informations provenant du texte ou du discours sous forme linéaire sont traitées en vue de la construction d'une «structure sémantique interne» (Deschênes 1988) ou une macrostructure (Denhière 1984) existant hypothétiquement en mémoire sous forme de réseau hiérarchisé de concepts reliés les uns aux autres.

Inversement des processus de compréhension, la production langagière demande des processus de linéarisation des structures plus ou moins hiérarchisées, stockées dans la mémoire (Kempen 1979; Denhière 1984; Fayol 1991). En interprétation consécutive, il s'agit, pour l'interprète, de reproduire la structure hiérarchisée représentant ce qu'il a compris du discours source.

Dans notre expérimentation, à quel moment les cartes de concepts et les résumés interviennent-ils dans les processus psychologiques de compréhension et de production durant l'interprétation? Regardons la figure 1:

La figure 1 suggère une ressemblance entre la structure de la carte et la hiérarchie des concepts en mémoire, produite par les processus de conceptualisation. Elle montre aussi la ressemblance entre la structure du résumé et celle, linéaire, du discours cible, produit des processus de linéarisation. Si ces deux outils d'intervention ont le même contenu mais non la même structure informative, comment leurs influences sur les processus de compréhension et de production en interprétation consécutive se ressemblent-elles ou se différentient-elles ? Avant de présenter les résultats répondant à cette question spécifique de recherche, nous décrivons brièvement comment a été menée l'expérimentation.

\section{L'EXPÉRIMENTATION}

Nous avons réalisé une recherche quasi expérimentale à la Faculté de français de l'École Supérieure de Langues Étrangères de Hanoi. L'échantillon comprend 61 étudiants de troisième et quatrième années. Dans cette école, la formation des interprètes et des traducteurs dure quatre ans. Les deux premières années sont réservées au perfectionnement du français. Les deux autres, la troisième et la quatrième, sont consacrées à l'apprentissage professionnel du métier de traducteur ou d'interprète (plutôt consécutif). 


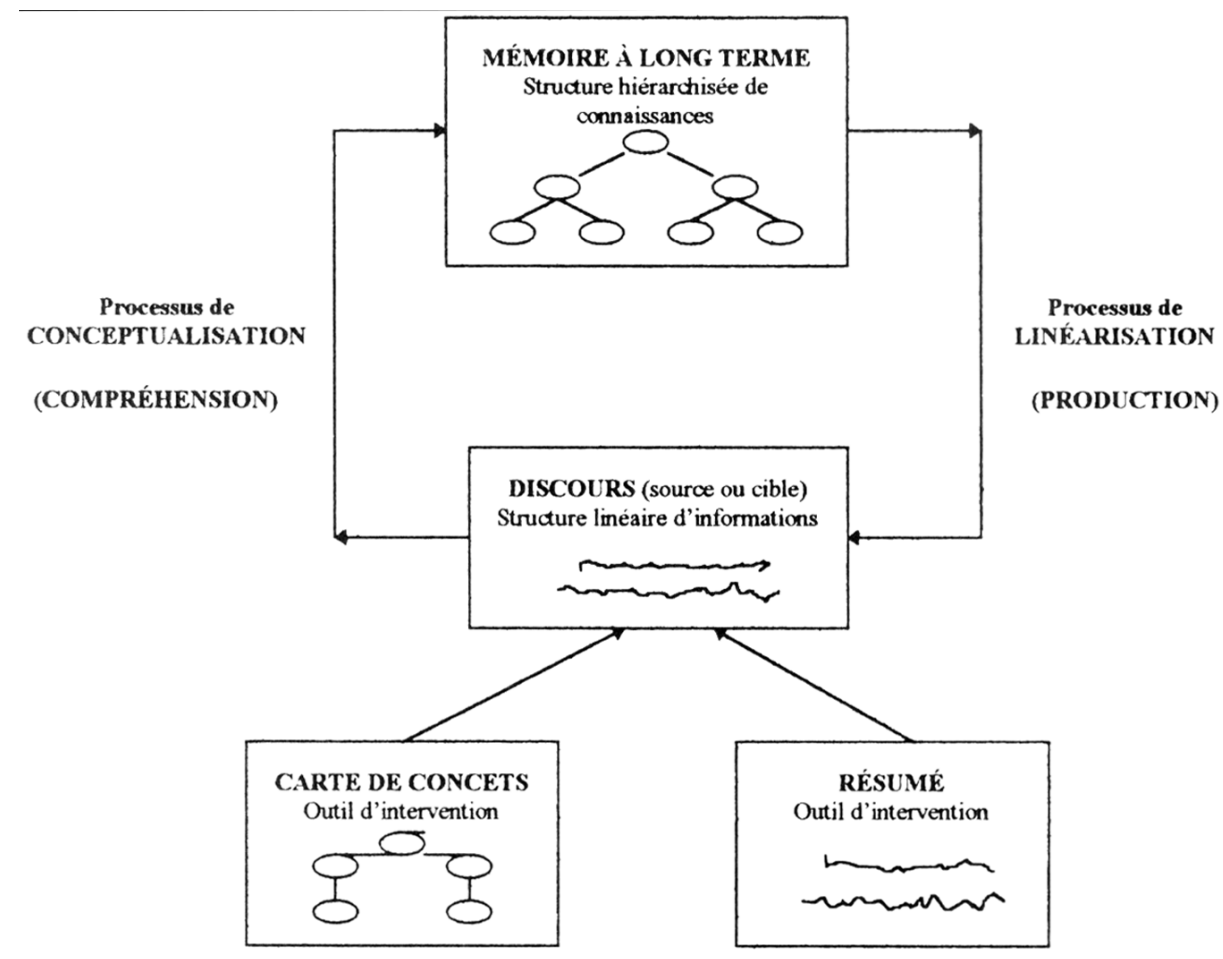

Figure 1

La carte de concepts et le résumé intervenant dans les processus d'interprétation ${ }^{3}$

Avant l'expérimentation proprement dite, les sujets ont participé à une séance d'initiation à la carte de concepts qui a duré 4 heures. Durant cette séance, ils ont appris ce qu'est une carte et comment lire une carte de concepts. Ils ont eu aussi l'occasion de faire une préexpérimentation tout en essayant d'interpréter deux passages de discours de deux minutes en utilisant une carte des macrostructures du discours à interpréter. En ce qui concerne le résumé, nous n'avons pas organisé de séance d'entrainement spéciale pour cette technique car les étudiants savent déjà comment construire et, évidemment, lire un résumé.

Au moment de l'expérimentation réalisée dans des laboratoires de langue, les sujets ont été répartis aléatoirement en trois groupes. Chaque sujet a dû interpréter trois discours, chacun à l'aide d'une technique différente : carte de concepts, résumé, ou prise de notes. Parmi ces trois techniques, c'est la première fois que la carte de concepts et le résumé étaient appliqués dans le processus d'interprétation. Il est à préciser ici que les sujets ne devaient pas construire eux-même la carte ou le résumé mais ceux-ci étaient fournis avant l'interprétation. Dans cette institution, la prise de notes est la technique habituelle que les sujets utilisaient déjà dans les heures d'exercices de l'interprétation en classe. Voici le schéma d'expérimentation de notre recherche : 


\begin{tabular}{|l|c|c|c|}
\hline & Groupe 1 & Groupe 2 & Groupe 3 \\
\hline Discours 1 & C & R & N \\
\hline Discours 2 & N & C & R \\
\hline Discours 3 & R & N & C \\
\hline
\end{tabular}

Tableau1

Le schéma d'expérimentation

Légende :

$\mathrm{C}$ : interprétation avec Carte de concepts

$\mathrm{R}$ : interprétation avec Résumé

$\mathrm{N}$ : interprétation avec Notes

Après la séance d'interprétation, 42 sujets ont participé à une entrevue semi-dirigée pendant laquelle ils ont répondu aux questions concernant l'influence de la carte de concepts et du résumé sur des variables telles que : la compréhension, la production, la prise de notes et l'état psychologique face à la présence de ces nouvelles techniques d'intervention. Ces réponses ainsi que les interprétations en vietnamien ont été enregistrées sur cassettes pour les analyses que nous présentons maintenant dans la section suivante.

\section{LES RÉSULTATS DE LA RECHERCHE}

Les données de la recherche ont été recueillies de deux sources. La première comprend les notes des interprétations évaluées par deux juges, professeurs en interprétation. Ces notes serviront d'input pour une analyse quantitative. La deuxième source est reliée aux réponses des sujets issues de l'entrevue semi-dirigée. Le corpus transcrit des enregistrements sur cassette sera utilisé pour une analyse qualitative. Enfin, une triangulation ${ }^{4}$ des résultats obtenus de l'analyse quantitative avec ceux de l'analyse qualitative sera faite dans le but de mieux valider les conclusions de la recherche.

\section{Les résultats de l'analyse quantitative}

L'analyse quantitative a été faite à partir des notes d'interprétation qui vont de 1 à 10 ; 1 est très mauvais et 10 est excellent. C'est une échelle de notation de laquelle les juges ont une grande expérience pratique. Les juges ignoraient quel sujet avait travaillé à l'aide de la carte, du résumé ou de la prise de notes. De cette analyse, nous tentons essentiellement de dégager les différences statistiquement significatives entre les groupes utilisant les différentes techniques d'intervention à savoir la carte de concepts, le résumé et la prise de notes. À cette étape de l'analyse, en raison des limites méthodologiques, on ne peut pas distinguer ce qui relève de la compréhension et ce qui relève de la production, à partir de l'interprétation en tant que produit (Dancette 1990).

Le test à distribution libre de Kruskal-Wallis a été appliqué, afin de comparer d'abord les résultats des sujets utilisant trois techniques d'intervention différentes, et ensuite, les résultats de trois groupes 1, 2, 3 et ceux de trois discours 1, 2, 3 (voir le Schéma d'expérimentation, tableau 1). Les résultats de cette analyse n'ont pas démontré de différence statistiquement significative entre les trois techniques d'intervention: $\mathrm{p}=$ $0,1573, \alpha=0,05$. Par curiosité scientifique et étant donné que le test Kruskal-Wallis ne permet pas de comparer les paires d'échantillons, nous avons utilisé un autre test non paramétrique, celui de Mann-Whitney. Grâce à ce dernier, les techniques ont été comparées par paires : carte et résumé, carte et notes, résumé et notes. Conformément aux résultats du 
test de Kruskal-Wallis, ce test n'a démontré aucune différence significative entre chaque paire de techniques. Cependant, il permet de mieux voir quelle paire de techniques a une différence se rapprochant du seuil de signification $\alpha=0,05$ :

$$
\begin{array}{lll}
\text { Carte }- \text { Notes : } & \mathrm{U}=1495,0 & \mathrm{p}=0,0612 \\
\text { Résumé - Notes : } & \mathrm{U}=1592,0 & \mathrm{p}=0,1691 \\
\text { Carte }- \text { Résumé } & \mathrm{U}=1786,5 & \mathrm{p}=0,7047
\end{array}
$$

\section{Les résultats du test $U$ de Mann-Whitney : comparaison de deux échantillons indépendants}

Selon les résultats ci-dessus, la différence entre les interprétations avec carte et sans autre technique d'intervention que la prise de notes usuelle est statistiquement la plus grande car la probabilité associée à la statistique $\mathrm{U}$ se rapproche le mieux du seuil de signification prévu. La différence entre les interprétations avec carte et celles avec résumé est statistiquement la plus faible.

Bien qu'il n'y ait pas de différence significative entre les techniques d'intervention, le test de Kruskal-Wallis montre qu'il existe des différences statistiquement fort significatives entre les résultats des groupes 1,2 et $3(\mathrm{p}=0,0004)$ ainsi qu'entre les résultats des discours 1,2 et $3(p=0,002)$. Ces différences significatives entre les groupes de sujets et les discours à interpréter suggèrent qu'il y a une inégalité entre les groupes et les discours. Les moyennes des rangs du groupe $2(112,66)$ et du discours $2(112,94)$ sont les plus fortes. Les moyennes des rangs du groupe $3(76,04)$ et du discours $3(73,68)$ sont les plus faibles. L'inégalité supposée du niveau des groupes (malgré une répartition aléatoire) et la variation du degré de facilité supposé des discours ont fort probablement réduit l'impact des techniques d'intervention. Ainsi, il faudrait d'autres recherches de type expérimental dans lesquelles les variables de groupe et de discours seraient mieux contrôlées pour mieux voir si la différence entre les techniques d'intervention est vraiment significative.

\section{Les résultats de l'analyse qualitative}

Les entrevues avec 42 sujets ont été analysées et ont dévoilé les influences différentes de la carte de concepts et du résumé sur deux variables principales : la compréhension du discours source, la production du discours cible, sur deux variables supplémentaires : la prise de notes et l'état psychologique des sujets au cours des interprétations pendant lesquelles ils utilisaient ces deux techniques.

Avant d'aborder ces influences différentes, provoquées par les structures propres à chaque technique (carte et résumé), il faut dire que ces outils d'intervention semblent représenter des avantages considérables pour la compréhension et la production pendant l'interprétation. À part deux sujets qui ont dit que la carte et le résumé les ont rendus trop dépendants, moins attentifs à l'écoute du discours source, 40 sujets (près de $95 \%$ ) ont répondu qu'ils préfèraient la carte et le résumé à l'absence de technique d'intervention autre que la prise de notes traditionnelle. Ce privilège en faveur de la carte et du résumé semble évident car ces deux outils permettent d'anticiper le contenu principal qui sera abordé dans le discours tandis qu'avec la prise de notes usuelle, les interprètes doivent se débrouiller tout seuls :

La carte et le résumé fournissent, tous les deux, les idées clés du discours à interpréter. La compréhension est ainsi bien facilitée (sujet B317) ${ }^{5}$.

Quant à la production interprétative, ces deux techniques de représentation du discours ont servi, pour presque tous les sujets, d'aide-mémoire permettant de ne pas manquer les idées importantes du discours : 
Heureusement, j'avais la carte et le résumé. J'ai une courte mémoire. En plus, mes notes ne sont pas toujours suffisantes pour bien interpréter. Au cours de la réexpression en vietnamien, j'ai dû regarder la carte et le résumé pour me souvenir de quelques idées oubliées (sujet C403).

Si nous allons maintenant un peu plus loin pour voir comment les structures différentes de la carte et du résumé ont participé au processus de conceptualisation (la conceptualisation est une production de compréhension), la carte semble perçue comme le facteur le plus actif pour la formation d'une structure globale du discours. Grâce à sa structure hiérarchique, la carte présente mieux visuellement la macrostructure du discours à interpréter. Les sujets semblent y voir clairement les idées clés, les relations entre celles-ci et le développement du discours, ce qui paraît moins évident avec un résumé :

La carte me montre dans quel sens le texte sera développé. Elle me donne l'orientation du texte tandis que dans le résumé, cette orientation n'est pas toujours claire (sujet A418).

La carte est un texte déjà analysé. On y voit clairement quelles sont les idées principales, quelles sont les idées supplémentaires. Avec le résumé, ce n'est pas comme ça. Il faut analyser ce texte pour en tirer ce qui est important (sujet B407).

Dans le résumé, je vois bien quel est le contenu du discours mais je ne vois pas très bien quel est l'ordre de présentation du discours. La carte fournit très bien ce contenu ainsi que cet ordre de présentation. Elle est comme un plan (sujet B317).

La réduction sémantique que représente la carte permet vraisemblablement, dans ce sens, une structuration plus rapide et plus efficace des informations.

Cependant, certains sujets (A402, A405, C404, C407) déclarent que la carte leur a causé des problèmes de compréhension tandis que le résumé n'a reçu aucune critique de ce type. Nous éliminons ici les difficultés de compréhension dues à une connaissance linguistique insuffisante et abordons seulement celles qui sont entraînées par l'utilisation des cartes. Les remarques sur les limites de la carte se concentrent essentiellement sur la partie «2 menaces» de la carte 1 et la complexité de la carte 3 . Les trois cartes utilisées dans l'expérimentation sont des cartes de surface 6 . Seule la partie «2 menaces» résumant trois derniers paragraphes du discours 1 représente la structure d'une carte profonde. Par exemple, pour la première menace, au lieu d'écrire «dématérialisation du travail», unité lexicale prise telle quelle du texte original, nous avons mis «mécanisation langagière», unité qui nomme mieux, à notre avis, la première menace pour le français. C'est cette mécanisation langagière qui est la cause directe mettant en péril le français tandis que la dématérialisation n'est qu'une cause indirecte, celle entraînant la précédente. Un autre exemple concernant la deuxième menace, celle de «l'élimination de l'ambiguïté langagière» qui a été présentée dans la carte comme suit (Ambiguïté langagière). Selon la carte, cette élimination de toute ambiguïté langagière vise à favoriser le travail des machines. Cette idée ne vient pas telle quelle du texte. Elle demande une compréhension approfondie du document car elle est abordée implicitement dans le dernier paragraphe où l'auteur parle de la vente d'une centrale nucléaire. Ces absences de correspondance avec la textualité ont mis quelques sujets (A402, A405) utilisant la carte 1 dans une situation embarrassante. Au moment de l'écoute du discours, pour la partie «2 menaces», ils n'ont plus trouvé trace, sur la carte, de ce que disait l'orateur. Une incertitude est apparue entre la compréhension de la carte et celle du discours, ce qui a freiné le processus d'analyse en désorientant l'attention du sujet :

Tout se passe bien jusqu'à cette partie-là [élimination de l'ambiguïté langagière]. Je trouve que ce que dit le discours ne correspond pas avec ce que dit la carte. Je jette un coup d'œil par ci, par là sur la carte pour essayer de trouver la logique de la carte. Je me demande si la carte est correcte ou si ce que j'ai entendu du discours est correct. Comme ça, je rate un passage du discours (sujet A405). 
Pour la carte 3, la principale difficulté est le nombre important d'informations ramassées dans la carte, qui donne l'impression aux sujets que cette carte est compliquée. Cette complexité s'explique par le fait que la carte suit de près les mots du texte 3 qui est plutôt de type descriptif, avec plusieurs énumérations et détails. Mais cette difficulté est vite résolue une fois que le sujet a bien saisi la suite logique de l'auteur, après l'écoute du discours.

Au niveau de la production, les opinions favorables ou défavorables se partagent entre la carte et le résumé, en concordance avec les types de fonctionnement variés des sujets. Ce qui est le plus apprécié dans la carte, à cette étape de linéarisation, est sa structure hiérarchique dans laquelle les idées sont présentées dans un ordre clair :

L'interprétation [la production] est beaucoup facilitée car la carte donne déjà un ordre de présentation. Je présente d'abord la première idée principale, ses idées supplémentaires, et puis la deuxième idée et ainsi de suite. J'ai l'impression que je m'exprime plus couramment avec la carte (sujet C402).

Cet ordre n'est pas toujours reconnu facilement avec le résumé. Il est plus difficile de s'exprimer avec le résumé car on n'y voit pas clairement l'ordre des idées (B316).

Un autre point fondamental, dans la production en interprétation, est le degré de liberté d'expression sans trahir le discours original (Gile 1990). Un bon nombre de sujets ont témoigné que la carte leur avait donné plus de liberté dans l'expression que le résumé. Ce phénomène s'explique par la structure sémantique réduite, très brève, de la carte. Dans la carte, on ne trouve que les mots clés reliés entre eux pour exprimer les idées essentielles du discours à interpréter. À partir de ces mots, l'apprenti-interprète a la liberté d'exprimer ces idées par des phrases formulées de façons différentes, pourvu que celles-ci reflètent fidèlement le vouloir-dire de l'orateur. Au contraire, le résumé avec ses phrases complètes a rendu certains sujets plus dépendants, lors du recours à cette technique, durant le processus de linéarisation :

Dans le résumé, les phrases sont déjà prêtes. Quand j'oublie une idée et je regarde dans le résumé pour me souvenir de cette idée et pour l'interpréter [la réexprimer], je suis dépendante de la façon d'exprimer du résumé (sujet B312).

La carte correspond mieux à la pensée [...] En travaillant avec le résumé, je ne suis plus moimême. Je dis les choses qu'un autre a écrites (sujet B316).

Il est fort possible que le sujet B316 ne soit pas conscient que le processus de production est un processus par lequel les informations reliées au discours à interpréter, organisées en structure hiérarchisée (comme la structure de la carte de concepts) sont linéarisées en une suite sonore. Il (B316) estime que «la carte correspond mieux à la pensée». Peut-on penser que la carte, par sa structure spéciale, favorise le transfert des informations structurales mises en mémoire en informations verbales linéaires ?

Toujours en ce qui concerne le degré de liberté d'expression que permettent la carte ou le résumé, à l'opposé des sujets qui se sentent dépendants du résumé et préfèrent s'exprimer dans leurs propres mots à l'aide de la carte, il existe des sujets qui préfèrent le résumé à la carte. Pour ceux-ci, il est plus difficile de s'exprimer avec la carte car elle ne se compose que de mots et non pas de phrases :

Dans le résumé, les phrases sont prêtes. Au moment de la réexpression, on n'a qu'à s'appuyer sur ces phrases. Les phrases prêtes sont plus faciles pour l'expression car nous travaillons toujours dans une situation d'interprétation phrase par phrase où on a plus de temps pour élaborer la langue cible (sujet B314).

Il est à préciser ici que d'habitude, pendant les heures d'exercice d'interprétation en classe, les sujets écoutent seulement une ou deux phrases du discours source et les inter- 
prètent en discours cible. Si l'interprétation de la première personne n'est pas satisfaisante, souvent, le professeur demande à une autre de donner sa version. Ainsi, les sujets ont toujours le temps de réfléchir plus longtemps, en plus de l'avantage lié à la longueur limitée du discours source. Dans notre expérimentation, c'était la première fois que les sujets essayaient d'interpréter des discours d'une telle longueur (500 mots, soit environ 4 minutes chaque). Cette durée est suffisamment longue, d'après Seleskovitch et Lederer (1989), pour que les sujets oublient les mots et ne travaillent que sur les idées. En plus, la carte ou le résumé sont trop simples s'ils sont construits seulement d'une ou de deux phrases. Malgré cette situation toute nouvelle mais qui peut fort probablement se produire dans un contexte professionnel, les sujets se sont débrouillés tant bien que mal. $95 \%$ des répondants ont affirmé lors des entrevues que la carte et le résumé sont utiles dans des cas pareils où le discours est long; les notes ne sont pas toujours suffisantes; la mémoire ne peut pas travailler de façon optimale à cause de la fatigue, du stress ou d'autres facteurs physiques ainsi que psychologiques.

Une autre influence exercée par les structures de la carte et du résumé que nous voulons aussi aborder concerne la visualisation des notes durant la restitution du discours cible. La réception visuelle (lecture) des notes déjà prises est un des trois composants de la deuxième phase du processus de production. Gile (1995 : 109) propose un modèle des efforts psychologiques requis dans l'interprétation consécutive comprenant deux phases :

$1^{\text {re }}$ phase $:$ Écoute + Mémoire à court terme + Prise de notes + Coordination des autres efforts $2^{e}$ phase : Mémoire à long terme + Lecture des notes + Production

L'organisation de ce qui est noté est aussi importante car elle facilite la lecture. Ce n'est pas sans raison que les cours d'interprétation comprennent aussi les heures de formation à la technique de prise de notes (noter les idées seulement, noter en diagonale, maîtriser les abréviations communes et personnelles...). Au moment de la reproduction du discours cible, la plupart des sujets de notre recherche ont réutilisé la carte et n'ont réutilisé le résumé que dans une moindre mesure. Ce fait se justifie par deux raisons. Premièrement, les sujets peuvent utiliser la carte comme leurs feuilles de notes car la structure de la carte permet de noter sur la carte elle-même. Les idées spécifiques peuvent être ajoutées à côté des idées générales se trouvant dans les cercles sur la carte. Le résumé n'offre pas cette condition car il est structurellement linéaire et il ne donne pas suffisamment d'espace entre les mots pour noter. Avec la technique de résumé, les sujets doivent faire une note à part comme d'habitude. Deuxièmement, même quand les sujets n'ajoutaient pas de notes sur la carte, la visualisation de la carte semblait beaucoup plus rapide que celle du résumé, nous a expliqué le sujet B407 :

Quand j'oublie une idée, je jette un coup d'œil dans la carte. C'est plus rapide et plus facile que regarder dans le résumé [...] Parce que dans la carte, je vois tout de suite les mots ou les idées avec leurs relations. Pour le résumé, c'est plus difficile. Ce sont des phrases qui se suivent, les unes après les autres. Il faut les relire pour me souvenir du contenu.

À part les questions portant sur la compréhension et la production, les deux processus principaux de l'interprétation consécutives, nous avons aussi posé aux sujets des questions reliées à l'impact de la carte de concepts et du résumé sur deux variables supplémentaires : la prise de notes et l'état psychologique du sujet face à ces nouvelles techniques, dans leur mise en pratique.

En ce qui concerne la prise de notes, comme nous l'avons mentionné plus haut, avec la technique de résumé, les sujets ont pris des notes à part tandis qu'avec la carte, ils ont créé une combinaison entre le contenu de la carte et les notes personnelles. Toutefois, certains sujets ont aussi pris des notes à part, même quand ils avaient une carte entre les mains. Les raisons sont multiples. Le sujet oubliait la consigne selon laquelle il pouvait 
prendre des notes sur la carte s'il le voulait; il trouvait qu'il n'y avait pas suffisamment d'espace sur la carte pour noter; étant donné que la carte est une technique nouvelle, il se sentait plus rassuré en prenant des notes à part et en utilisant la carte seulement dans le but de confronter des informations ou d'anticiper l'oubli.

Quant à l'état psychologique, à part deux sujets (5\%) qui n'aimaient pas la carte et le résumé, le reste des sujets ont affirmé qu'ils se sentaient plus rassurés en recevant des techniques d'aide car ils savaient au moins de quoi il s'agissait dans le discours. Ces deux sujets préféraient travailler sans carte ni résumé parce qu'ils avaient l'impression qu'ils devenaient «dépendants» des techniques d'intervention et qu'ils ne pouvaient plus travailler de façon «autonome».

\section{La triangulation des résultats quantitatifs et qualitatifs}

Nous terminons cette présentation des résultats de la recherche par une triangulation des résultats de l'analyse quantitative avec ceux de l'analyse qualitative. Bien que l'analyse quantitative ne montre pas de différence statistiquement significative entre les trois techniques d'aide à l'interprétation (carte, résumé, notes), les perceptions des sujets qui les ont utilisées mettent en lumière, qualitativement, des influences différentes que ces techniques exercent sur leur compréhension et leur production langagières. Parmi les interviewés, 29 sujets $(69 \%)$ ont répondu que la carte les a davantage aidé, 9 sujets $(21 \%)$ préféraient le résumé, 2 sujets $(5 \%)$ trouvaient que la carte et le résumé étaient égaux et 2 sujets (5\%) préféraient travailler sans technique dintervention autre que la prise de notes traditionnelle. Si nous confrontons ces pourcentages des jugements des sujets aux résultats du test U de Mann-Whitney, nous voyons qu'il y a deux convergences majeures entre ces données quantitatives et qualitatives. Premièrement, selon l'analyse quantitative, la différence entre Carte et Notes est statistiquement plus grande que celle entre Résumé et Notes. Dans ces deux paires de techniques, les moyennes des rangs de la carte et du résumé sont toujours supérieures à celles des notes. Ceci suggère que la carte et, après elle, le résumé apportent aux sujets une meilleure aide que les notes personnelles. Ce classement du degré d'utilité des techniques selon l'analyse quantitative correspond aux appréciations positivement supérieures en faveur de la carte et, ensuite, du résumé par rapport aux notes, selon l'analyse qualitative.

Deuxièmement, l'hypothèse d'une inégalité des discours à interpréter, issue de l'analyse quantitative est confirmée par les jugements objectifs des sujets. La plupart des répondants ont évalué que le discours 2 est le plus facile. Cette perception est conforme aux résultats quantitatifs qui démontrent que la moyenne des interprétations du discours 2 est la plus élevée. Pour les discours 1 et 3 qui restent, deux positions opposées s'égalisent : certains disent que le discours 1 est le plus difficile, d'autres prétendent que c'est plutôt le discours 3. Les jugements de facilité ou de difficulté des discours à interpréter se justifient par plusieurs raisons : familiarité du thème traité, mots nouveaux, clarté des arguments de l'orateur, longueur du discours, état de santé du sujet.

L'opinion favorable accordée par la majorité des sujets en faveur de la carte de concepts comme aide à l'interprétation consécutive ne signifie pas que la carte puisse être appliquée immédiatement, par tout le monde et à toute situation professionnelle. L'efficacité de la carte dépend de plusieurs facteurs : type de fonctionnement du sujet, son aptitude pour l'expression orale (capacité de s'exprimer à partir des mots), type de carte (carte descriptive ou argumentative, carte de surface ou carte profonde). Il est à noter aussi que la carte ou le résumé ne permettent pas de résoudre les problème linguistiques. Si le sens d'un mot important n'est pas connu, la carte ou le résumé n'éclaircissent pas mieux le contenu à interpréter. La carte et le résumé ne sont pas non plus des outils magiques capables d'amoindrir les difficultés de compréhension orale dans tous les cas. Si le sujet ne com- 
prend pas une partie de la carte ou du résumé et si à l'écoute du discours, il ne trouve toujours pas la signification ou la suite d'idées de cette partie, rien ne peut l'aider. Le sujet devra laisser ce passage ou son interprétation deviendra une sorte de charabia.

L'application de la carte de concepts dans l'apprentissage et dans l'enseignement de l'interprétation consécutive demanderait plus d'heures d'entraînement avec des types de carte plus variés, pour que les sujets maîtrisent mieux la lecture des cartes conceptuelles. Si l'on souhaite former les étudiants à la création de cartes, il faudrait, à notre avis, au moins 10 heures d'entraînement. La création de la carte de concepts par l'étudiant luimême permettrait des applications intéressantes :

- La carte peut être utilisée pour contracter les documents distribués pour une séance d'interprétation.

- Avant une séance d'interprétation concernant un sujet quelconque, l'étudiant peut faire un brainstorming de tout ce qu'il sait du sujet, à l'aide d'une carte de concepts récapitulative. Cette stratégie de sensibilisation pourrait permettre à l'étudiant d'anticiper le sujet et de bien se préparer au travail.

- L'interprète peut prendre note du discours à interpréter sous forme d'une carte. Il est évident que cette carte ne peut pas être une carte perfectionnée comme celle des experts car cette dernière demande du temps et une analyse en profondeur du texte. Cependant, une carte simple avec des idées clés et les flèches reliant ces idées sont toujours faisables. Nous avons vu une sorte de carte simple dans laquelle les idées sont numérotées et reliées par des flèches sur la feuille de notes d'un sujet. Cette étudiante a reconnu qu'elle s'inspirait de la carte pour son interprétation, et il lui semblait que les notes devenaient ainsi plus claires pour la lecture.

- La conscience permanente de la construction d'une carte de concepts pourrait aussi contribuer à une meilleure compréhension du discours car une telle construction exige que l'étudiant se pose tout le temps une série de questions : quelles sont les idées essentielles de ce passage ? Quelle est la relation logique entre ces idées ?... Dans ce cas, la carte favoriserait la métacognition, c'est-à-dire le contrôle du processus d'analyse du discours à interpréter.

L'usage des cartes de concepts promet de belles perspectives pour la recherche sur l'apprentissage et l'enseignement de l'interprétation consécutive. Sur ce terrain à peine défriché de l'interprétation assistée par la cartographie des concepts, la présente recherche constitue une première, qui mérite de nombreux approfondissements.

\section{Notes}

1. Nous remercions l'Agence Canadienne de Développement International qui a financé la présente recherche.

2. Dans cet article, le mot «carte» désigne toujours «carte de concepts».

3. Pour créer cette figure, nous nous sommes inspirés de Novak et Gowin (1984).

4. «La triangulation est une stratégie qui vise à valider les résultats d'une recherche, grâce à la multiplication des méthodes d'analyse et la confrontation des résultats obtenus par des moyens différents» (Tochon $1991: 86$ ).

5. La traduction est de nous.

6. Ahlberg (1991) distingue deux types de cartes de concepts pour l'analyse des textes: carte de surface et carte profonde. La carte de surface se situe au niveau des mots du texte tandis que la carte profonde représente notre interprétation de ce qui est vraiment dit dans le texte.

\section{RÉFÉRENCES}

AHLBERG, Mauri (1991): «Concept Maps and Argumentation Analysis in the Analysis of School Texts», Article présenté lors du Congrès sur l'analyse de textes pédagogiques et l'analyse de contenu, Suède, Harnosand, Université de Joensuu, novembre. 
BRETON, Jacques (1991) : «La schématisation des concepts : un instrument de développement des habiletés conceptuelles au collégial», Pédagogie collégiale, vol. 4, n 3, fév., pp. 18-23.

BUZAN, Tony (1974) : Une tête bien faite, Paris, Organisation.

CLIBURN, Joseph W. Jr. (1986) : «Using Concept Map to Sequence Instructional Materials», Journal of College Science Teaching, vol. 15, n 4, Feb., pp. 377-379.

CLIBURN, Joseph W. Jr. (1990) : «Concept Maps to Promote Meaningful Learning», Journal of College Science Teaching, vol. XIX, ${ }^{\circ} 4$, pp. 212-217.

DANCETTE, Jeanne (1990) : Étude réflexive et expérimentale du processus de compréhension dans l'activité de traduction, Montréal, Québec, Université de Montréal, Thèse de doctorat présentée au Département de linguistique et philologie.

DANCETTE, Jeanne (1992) : «Modèles sémantique et propositionnel de l'analyse de la fidélité en traduction», Meta, vol. 37, n 3, pp. 440-449.

DANCETTE, Jeanne (1994) : «De la coïncidence accidentelle à la coïncidence dirigée — la traduction comme savoir-faire et les modèles logico-sémantiques qui en rendent compte», Meta, vol. 39, n 3, pp. 439-445.

DENHIÈRE, Guy (1984) : Il était une fois... Compréhension et souvenirs de récits, Lille, Presses Universitaire de Lille.

DESCHÊNES, André-Jacques (1988) : La compréhension et la production de textes, Sillery (Québec), Presses de l'Université du Québec.

FAYOL, Michel (1991) : «From sentence production to text production : investigating fundamental processes», European Journal of Psychology of Education, vol. VI, n 2, pp. 101-119.

FOURNIER, J. et R. DUTERTRE (1982) : Le résumé de textes par l'exemple, Paris, Roudil Éditeur.

GILE, Daniel (1990) : «La traduction et l'interprétation comme révélateurs des mécanismes de production et de compréhension du discours», Meta, vol. 35, n 1. pp. 20-30.

GILE, Daniel (1995) : Regards sur la recheche en interprétation de conférence, Lille, Presses Universitaires de Lille.

KEMPEN, G. A. M. (1979) : «La mise en paroles, aspects psychologiques de l'expression orale», Études de linguistique appliquée, $\mathrm{n}^{\circ} 33$, janv.-mars, pp. 18-28.

KINTSCH, W. et T. A. van DIJK (1975) : «Comment on se rappelle et on résume des histoires», Langues, $\mathrm{n}^{\circ} 40$, déc., Didier, Larousse.

KINTSCH, W. et T. A. van DIJK (1978): «Toward a model of text comprehension and production», Psychological Review, vol. 85, n 5, Sept., pp. 363-394.

LINDSAY, P.H. et D.A. NORMAN (1980) : Traitement de l'information et comportement humain, Montréal, Qc, Études vivantes.

NAIDU, Som (1990) : «Concept mapping, Student workbook», Développé comme une partie de matériaux de recherche pour le degré de doctorat à l'Université Concordia (Canada), ERIC-ED 329247.

NOVAK, J. D. (1981) : «The Use of Concept Mapping and Gowin's "V" Mapping Instructional Strategies in Junior High School Science», Report of the Cornell University "Learning how to learn" Project, Ithaca, New York, Cornell University.

NOVAK, J. D. and D. B. GOWIN (1984) : Learning how to learn, New York, Cambridge University Press.

NGUYEN, Phuong T. C. et François Victor TOCHON (1995) (sous presse) : «Influence de la carte de concepts sur les processus de compréhension et de production orales propres à l'interprétation consécutive, une nouvelle orientation de recherche», Puente, Lima, Pérou, UNIFE.

PANKRATIUS, William J. and Thomas Mark KEITH (1987) : «Building an Organized Knowledge Base : Concept Mapping in Secondary School Science», Paper presented at the Annual Meeting of the National Science Teachers Association (35th, Washington, DC, March 26-29, 1987), ERIC-ED 280720.

SELESKOVITCH, Danica et Marianne LEDERER (1989) : Pédagogie raisonnée de l'interprétation, BruxellesLuxembourg, Didier Érudition, coll. «Traductologie», $n^{\circ} 4$.

STICE, Carole F. and Marino C. ALVAREZ (1986) : «Hierarchical Concept Mapping : Young Children Learning How to Learn (A viable Heuristic for the Primary Grades)», Center of Excellence, Basic Skills for the Disavantaged, Reading/Writing Component Report No. 5, ERIC-ED 274946.

STICHT, T. G., L. G. BECK, R. N. HAUKE, G. M. KLEINAM and J. H. JAMES (1974) : Auding and Reading: A Developmental Model, Alexandria, VA, Human Resources Research Organisation (HumRRO).

TARDIF, Jacques (1992) : Pour un enseignement stratégique, Montréal, Éditions Logiques.

TOCHON, François Victor (1990) : «Les cartes de concepts dans la recherche cognitive sur l'apprentissage et l'enseignement», Perspectives documentaires en éducation, $\mathrm{n}^{\circ} 21$, pp. 86-105.

TOCHON, François Victor (1991) : L'enseignement stratégique : transformation pragmatique de la connaissance dans la pensée des enseignants, Toulouse, Éditions universitaires du Sud.

TROCHIM, William M. (1989) : «An introduction to concept mapping for planning and evaluation. Special Issue : Concept mapping for evaluation and planning», Evaluation and Program Planning, vol. 12, $\mathrm{n}^{\circ}$ 1, pp. 1-16.

VALENTINE, Kathleen (1989) : «Contributions to the theory of care. Special Issue : Concept mapping for evaluation and planning», Evaluation and Program Planning, vol. 12, n 1, pp. 17-23. 
ANNEXE 1

\section{LE DISCOURS 1, LA CARTE DE CONCEPTS 1 ET LE RÉSUMÉ 1}

\section{Discours 1}

\section{LE FRANÇAIS EN DANGER DE MORT}

Aujourd'hui, je vous parlerai du danger causé par la révolution informatique pour la langue française.

Nous sommes à la veille d'un bouleversement technologique important comme l'a été autrefois l'invention de l'écriture, ou plus récemment l'imprimerie. Les langues naturelles réagissent comme des êtres vivants; comme les espèces animales, elles évoluent sous la pression de leur environnement et subissent la loi de la sélection de Darwin. Je peux vous donner deux exemples. Quand l'imprimerie est apparue dans le Maghreb, l'arabe a été choisi comme la langue de l'imprimé, bien que le berbère fût (soit) pratiqué plus couramment. Le résultat est que le berbère a régressé à l'état de dialecte, tandis que l'arabe devenait une langue internationale. C'est le même cas pour le latin. Au temps de la bibliothèque d'Alexandrie, le latin a été remplacé par le grec malgré sa puissance parce que le grec était plus adapté à la nouvelle pensée scientifique née avec l'essor mécanique, astronomique...

Je viens de parler de la régression du berbère et du latin causée par le progrès technique et scientifique. J'aborde, maintenant, un cas concret, le cas du français devant la révolution informatique, ce qui est d'ailleurs le contenu principal de ce séminaire. Dans cette révolution, d'où viennent les menaces pour le français ? À mon opinion, actuellement, deux forces sont à l'œuvre et qui vont façonner le paysage linguistique.

La première de ces forces, c'est la dématérialisation du travail. Il y a seulement un siècle et demi, $90 \%$ de la puissance de travail, dans les champs ou dans les usines, étaient essentiellement musculaires. Les gens travaillaient plutôt avec leurs muscles. Aujourd'hui, plus de $70 \%$ des gens travaillent sur l'écrit : ils ne font que traiter et échanger des messages d'information, sur papier ou sur écran d'ordinateur. Leur outil de travail, c'est la langue. Il y a donc un appel à la mécanisation de ces opérations : c'est le rôle des systèmes de traitement de texte.

La deuxième force qui met en jeu l'avenir du français, c'est que dans la communication scientifique, technique, commerciale ou financière, on a besoin d'éliminer toute ambiguïté. Les termes doivent être exacts, pour éviter une interprétation incertaine. Or, comme vous le savez, les langues naturelles ont toujours un peu de jeu, de mou. C'est cette ambiguïté qui fait que la conversation est possible entre deux personnes d'opinions différentes : l'un va vouloir convaincre l'autre, le séduire. S'il n'y a pas une certaine équivoque, il n'y a pas de négociation possible.

L'élimination de toute ambiguiité langagière menace aussi la richesse littéraire, poétique d'une langue. C'est cette richesse qui donne à la langue sa puissance d'évocation, sa force imaginaire. Mais imaginez si vous vendez une centrale nucléaire à un pays étranger, vous fournissez aussi des consignes ou des instructions d'installation, d'utilisation et de maintenance... Tout cela représente des tonnes d'équivalent papier qui vont être stockées dans des mémoires informatiques. Il ne s'agit pas d'une seule phrase ou d'un seul mot à interpréter. Il faut que les messages soient contrôlés par une machine. C'est la première fois dans l'histoire que l'on trouve la nécessité de travailler la langue pour la débarrasser de toute ambiguïté. On comprend le choc que cela a causé pour les littéraires.

Extrait et adaptation de l'article «Le français en danger de mort», Le nouvel observateur, $\mathrm{n}^{\circ}$ 1494, du 24 au 30 juin 1993 
La carte de concepts du texte 1

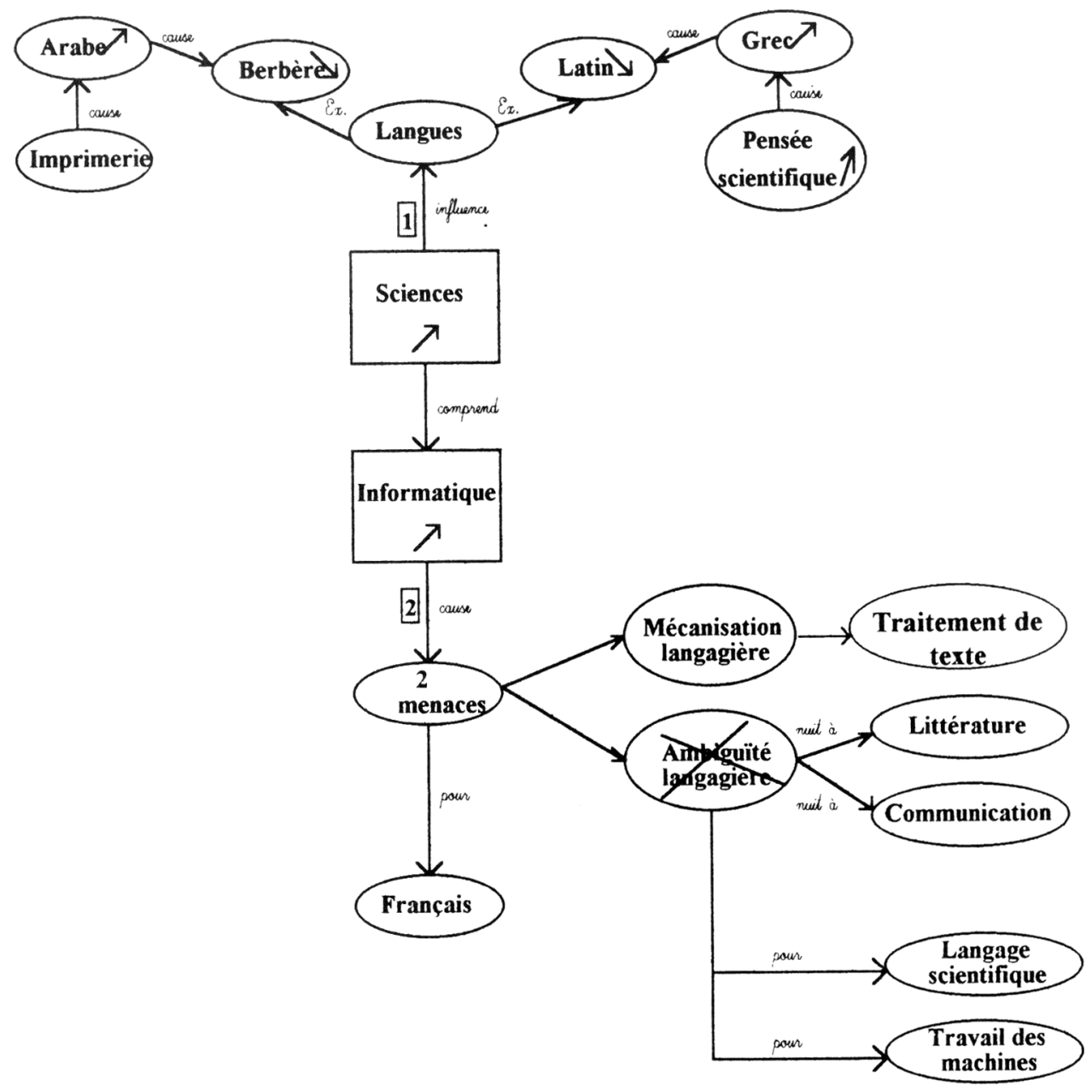

\section{Le résumé du discours 1}

Si les progrès techniques et scientifiques pouvaient condamner autrefois certaines langues comme le berbère et le latin, aujourd'hui, la révolution informatique met en danger le français par deux forces.

Premièrement, la dématérialisation du travail en faveur de l'écrit fait de la langue un outil de travail majoritaire. Il y a donc un appel à la mécanisation langagière par le moyen des systèmes de traitement de texte.

Deuxièmement, c'est l'élimination de toute ambiguïté langagière pour assurer l'exactitude des termes dans la communication scientifique, technique, commerciale ou financière. Une telle élimination rend la négociation impossible et menace la richesse littéraire, poétique d'une langue. 